\title{
Comment on the paper 'Prospective pediatric study comparing glomerular filtration rate estimates based on motion-robust dynamic contrast-enhanced magnetic resonance imaging and serum creatinine (eGFR) to 99mTc DTPA'
}

\author{
Diego De Palma ${ }^{1}$ \\ Received: 17 April 2020 / Revised: 12 May 2020 / Accepted: 19 October 2020 / Published online: 19 November 2020 \\ (C) Springer-Verlag GmbH Germany, part of Springer Nature 2020
}

\section{Dear Editors,}

I would like to comment on the paper "Prospective pediatric study comparing glomerular filtration rate estimates based on motion-robust dynamic contrastenhanced magnetic resonance imaging and serum creatinine (eGFR) to 99mTc DTPA," by Kurugol et al. [1].

I would like to congratulate the authors for the welldesigned work and their excellent results, but there were some parts that, in my opinion, need a better definition, especially dealing with the reference method of glomerular filtration rate (GFR) estimate with $99 \mathrm{mTc}$ diethylenetriamine pentaacetic acid (DTPA).

This method is described in a technical guideline of the European Association of Nuclear Medicine (EANM) [2], in which the suggested methodology and administered activities differ from those reported by the authors. In this guideline the method recommended requires only one blood sample, taken between $110 \mathrm{~min}$ and $130 \mathrm{~min}$ after administration (that obviates for the greater part of blood sample timing problems) and implies a lower exposure, keeping it far below the "alarm threshold" of $1 \mathrm{mSv}$ set by International Commission on Radiological Protection.

It must be said, also, that affirming that one of the advantages of MRI in respect to radionuclide imaging is "measuring the differential GFR of each kidney" is unfair, because with the administered activities reported by the authors it is

Diego De Palma

diego.depalma61@gmail.com

1 Nuclear Medicine Unit, Circolo Hospital and Macchi Foundation, ASST-Settelaghi, viale L. Borri 57, 21100 Varese, Italy possible to perform a high-quality renal dynamic scan, obtaining the relative differential renal function and then the differential GFR. In this setting, radiation exposure still remains well below $1 \mathrm{mSv}$.

Other advantages of estimating GFR with 99mTc DTPA are that it can be calculated also in children with poor renal function (see the EANM guideline), that radiopharmaceuticals have no toxicity or allergic side effects, and no sedation is normally necessary for the simple GFR estimate or for performing a full dynamic renal scan.

Finally, DTPA GFR is cheaper than MR-GFR; for example, its reimbursement according to Italian National Health Service rules is $160 €$ against $250 €$ for renal contrast-enhanced MRI.

Then, it seems that GFR estimate by MRI does not have such an advantage in respect to the radionuclide method.

\section{Compliance with ethical standards}

Conflicts of interest None

\section{References}

1. Kurugol S, Afacan O, Lee RS et al (2020) Prospective pediatric study comparing glomerular filtration rate estimates based on motionrobust dynamic contrast-enhanced magnetic resonance imaging and serum creatinine (eGFR) to ${ }^{99 m}$ TcDTPA. Pediatr Radiol 50:698-705

2. Piepsz A, Colarinha P, Gordon I et al (2001) Guidelines for glomerular filtration rate determination in children. Eur J Nucl Med 28: BP31-BP36

Publisher's note Springer Nature remains neutral with regard to jurisdictional claims in published maps and institutional affiliations. 